\title{
Fine Needle Aspiration of Primary Mediastinal Synovial Sarcoma: Cytomorphologic, Immunohistochemical, and Molecular Study
}

Cheng Cheng Huang, M.D., Ph.D., Claire W. Michael, M.D., and

Judy C. Pang, M.D. ${ }^{*}$

The cytologic diagnosis of synovial sarcoma (SS) can be difficult when it occurs in unusual locations, atypical age groups, and/or have unusual morphology. We report a case of primary mediastinal SS in a 65-year-old male with a long smoking history who presented with increasing shortness of breath and was found to have a $14.2 \mathrm{~cm}$ mediastinal mass. Smears from the endobronchial ultrasound guided fine needle aspiration of the mass were moderately cellular consisting of loosely cohesive clusters, some of which demonstrated nuclear molding, and dispersed single cells. The relatively uniform tumor cells had a high nuclear-to-cytoplasmic ratio, finely granular chromatin, and inconspicuous nucleoli. Some of the single cells had spindled morphology with unipolar wispy tails and naked nuclei. Based on the clinical presentation and the cytomorphologic features, our initial differential diagnoses included atypical carcinoid, small cell carcinoma, basaloid squamous cell carcinoma, sarcoma, and lymphoma. Immunohistochemical studies on the cell block sections revealed that the tumor cells were focally positive for cytokeratin and diffusely positive for CD56, while negative for CD45, synaptophysin and chromogranin. Ultimately, an immunohistochemical stain for TLE-1 demonstrated diffusely strong nuclear positivity and molecular studies showed the presence of the $t(X ; 18) S Y T / S S X 1$ translocation confirming the diagnosis of SS. In this report, we describe the cytomorphologic features of SS, its diagnostic pitfalls, and potential mimics in the mediastinum. Diagn. Cytopathol. 2014;42:170-176. @ 2012 Wiley Periodicals, Inc.

Key Words: synovial sarcoma; mediastinum; fine needle aspiration; cytology

Synovial sarcoma (SS) is one of the common soft tissue sarcomas occurring predominantly in the extremities around the large joints of adolescents and young adults. ${ }^{1-3}$ Although the majority of SS arise in the lower extremity, a

Department of Pathology, University of Michigan, Ann Arbor, Michigan

*Correspondence to: Judy C. Pang, M.D., Department of Pathology, University of Michigan, 1500 E. Medical Center Dr., Ann Arbor, MI 48109, USA. E-mail: jcpang@med.umich.edu

Received 17 May 2012; Accepted 16 July 2012

DOI $10.1002 /$ dc.22912

Published online 31 August 2012 in Wiley Online Library

(wileyonlinelibrary.com) significant number arise from other anatomic sites such as abdominal wall, ${ }^{4}$ head and neck, ${ }^{5}$ retroperitoneum, ${ }^{6}$ lung, ${ }^{7}$ and mediastinum. ${ }^{8-10}$ The cytomorphology of fine needle aspiration (FNA) biopsies from primary SS of various anatomic sites has been previously described. ${ }^{11,12}$ Primary mediastinal SS is very rare. ${ }^{8}$ To our knowledge, the cytomorphology and its potential mimics in the lung and mediastinum, especially carcinomas, have not been well described in the literature to date.

We report the FNA of an unusual SS occurring in the mediastinum in a 65-year-old man with a long smoking history. We discuss the potential diagnostic pitfalls due to its overlapping cytologic features and immunohistochemical-staining pattern with other neoplasms commonly encountered in FNAs of the mediastinum.

\section{Case History}

A 65-year-old male with a history of chronic obstructive pulmonary disease and smoking since he was 8 years of age presented with increasing shortness of breath over a 1-year period. Computed tomography (CT) scan showed a heterogeneous mass with necrosis measuring $14.2 \mathrm{~cm}$ in the left hilum of the mediastinum and extending to the left upper lobe of the lung. An endobronchial ultrasound showed near complete obliteration of the left main bronchus.

\section{Materials and Methods}

An endobronchial ultrasound guided FNA of the mediastinal mass was performed by a pulmonologist with cytology present for on-site evaluation. The aspirated material was expressed on the slides and smears were made. All of the slides were air-dried. Approximately half of the slides were stained with Diff-Quik for on-site evaluation, while the remaining slides were rehydrated in saline, alcohol fixed, and then stained with Papanicolaou stain. A 


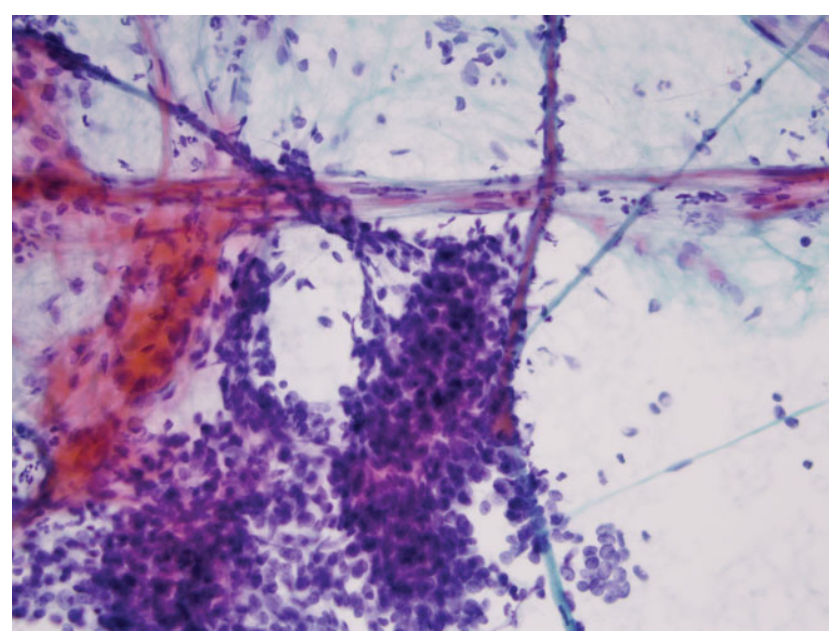

Fig. 1. Cell clusters intermingled with branches of delicate capillaries were occasionally seen. Papanicolaou stain, $400 \times$. [Color figure can be viewed in the online issue, which is available at wileyonlinelibrary.com.]

portion of the aspirated material was rinsed in RPMI media and a cell block was prepared with the thrombin clot method. The pellet was fixed in $10 \%$ buffered formalin and embedded in paraffin. Hematoxylin and eosin (H\&E) stains and immunohistochemical (IHC) studies were performed on the paraffin-embedded cell block using standard laboratory methods. The antibodies used for IHC studies included cytokeratin cocktail, TTF-1, CD56, synaptophysin, chromogranin, CD45, Ki-67, p63, CK5/6, and TLE-1. The $\mathrm{t}(\mathrm{X} ; 18) \mathrm{SYT} / \mathrm{SSX}$ translocation assay was performed by using the protocol as described previously. ${ }^{13}$ Briefly, a multiplex reverse transcription polymerase chain reaction (RT-PCR) was performed on the RNA that was extracted from formalin fixed, paraffin embedded tissue using primers that are differentially labeled with fluorochrome dyes on their $5^{\prime}$ termini. The forward primer targets exon 10 of the SYT gene. One reverse primer targets a sequence common to exon 5 in all SSX gene family members, while additional primers target gene-specific regions of exon 5 of the SSX1 or SSX2 gene. The different sizes of the PCR products between the SSX1 and SSX2 fusion genes can be detected and sized by capillary electrophoresis. The integrity of the extracted RNA is evaluated in the same reaction mixture by amplification of a housekeeping gene (GAPDH) transcript.

\section{Microscopic Findings}

The aspirate smears were moderately cellular consisting of loosely cohesive cell clusters admixed with dispersed single cells. The cell clusters demonstrated a fascicular growth pattern with irregular borders. Branches of delicate vessels intermingled with the cell clusters were occasionally identified (Fig. 1). The cell clusters were composed of relatively uniform cells, which were small to

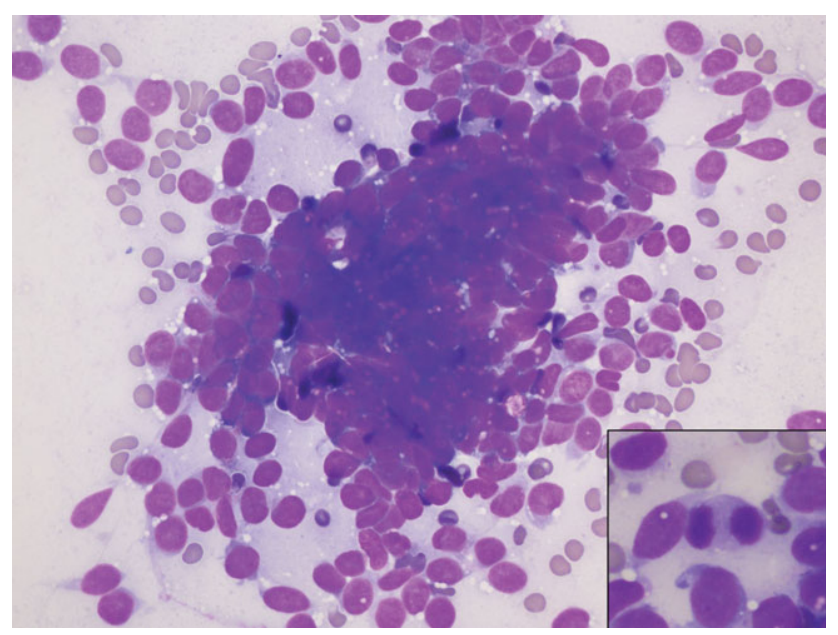

Fig. 2. Cell clusters are composed of small to medium-sized uniform cells with very scant cytoplasm. Nuclear molding is occasionally present. Mitotic figures are not uncommon (insert). Diff-Quik stain, 600×. [Color figure can be viewed in the online issue, which is available at wileyonlinelibrary.com.]

medium in size, round to oval with scant cytoplasm and very high nuclear to cytoplasmic (N/C) ratio. Nuclear molding was occasionally seen (Fig. 2). Occasional mitotic figures were identified (Fig. 2, insert). Trabecular and pseudoacinar architectures were focally present (Fig. 3, panel A). The nuclei had finely granular or powdery chromatin with small inconspicuous nucleoli (Fig. 3, panel A). Apoptotic bodies and tumor necrosis were not apparent. The single dispersed cells were spindled in shape with predominantly unipolar wispy tails. Naked nuclei were also frequent (Fig. 3, panel B). H\&E stained sections of the cell block revealed small round blue cells intermingled with vessels. Mitotic figures were readily identified.

\section{Immunohistochemical and Molecular Studies}

The IHC studies performed on the cell block demonstrated diffusely positive nuclear staining for TLE-1 (Fig. 4, panel B), membranous staining for CD56 (Fig. 4, panel C), and focal positivity for cytokeratin (Fig. 4 panel D). The malignant cells were negative for CD45, cytokeratin 5/6, p63, TTF-1, chromogranin, and synaptophysin. The proliferation rate was estimated by $\mathrm{Ki}-67$ to be $\sim 30 \%$. Molecular studies showed the presence of a $t(X$; 18) SYT/SSX1 translocation (Fig. 5).

\section{Discussion}

SS is a well described malignancy that most often arises in the soft tissues of the extremities of young adults. The diagnosis can be challenging when SS occurs in unusual locations, atypical age groups, and/or have unusual morphology. Our patient had a long smoking history and a primary mediastinal SS, which is rare, with only 15 cases 
HUANG ET AL.

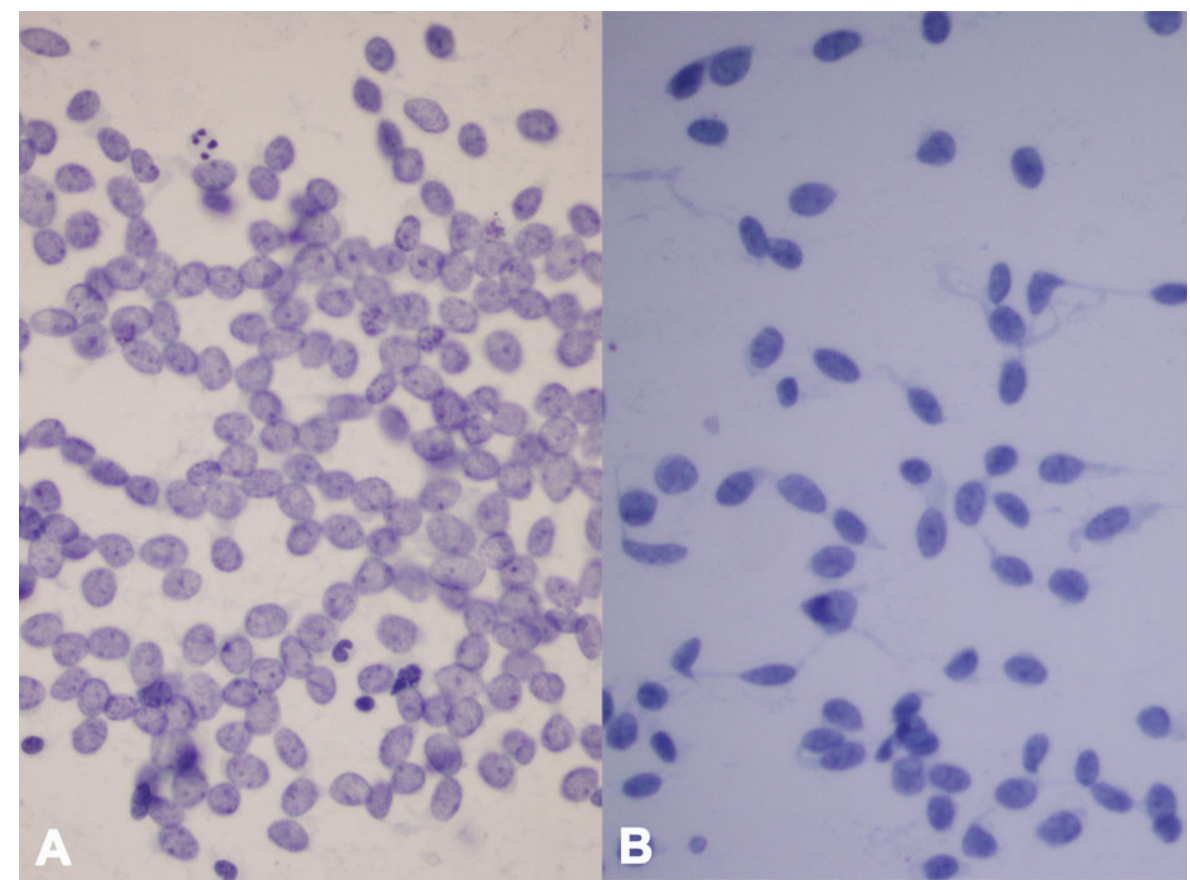

Fig. 3. The nuclei are round to oval with finely granular or powdery chromatin and small or inconspicuous nucleoli. Trabecular and pseudoacinar structures are focally present (A). Spindle shaped cells with elongated nuclei and fine wispy unipolar cytoplasmic tails are often seen (B). Papanicolaou stain, $600 \times$. [Color figure can be viewed in the online issue, which is available at wileyonlinelibrary.com.]

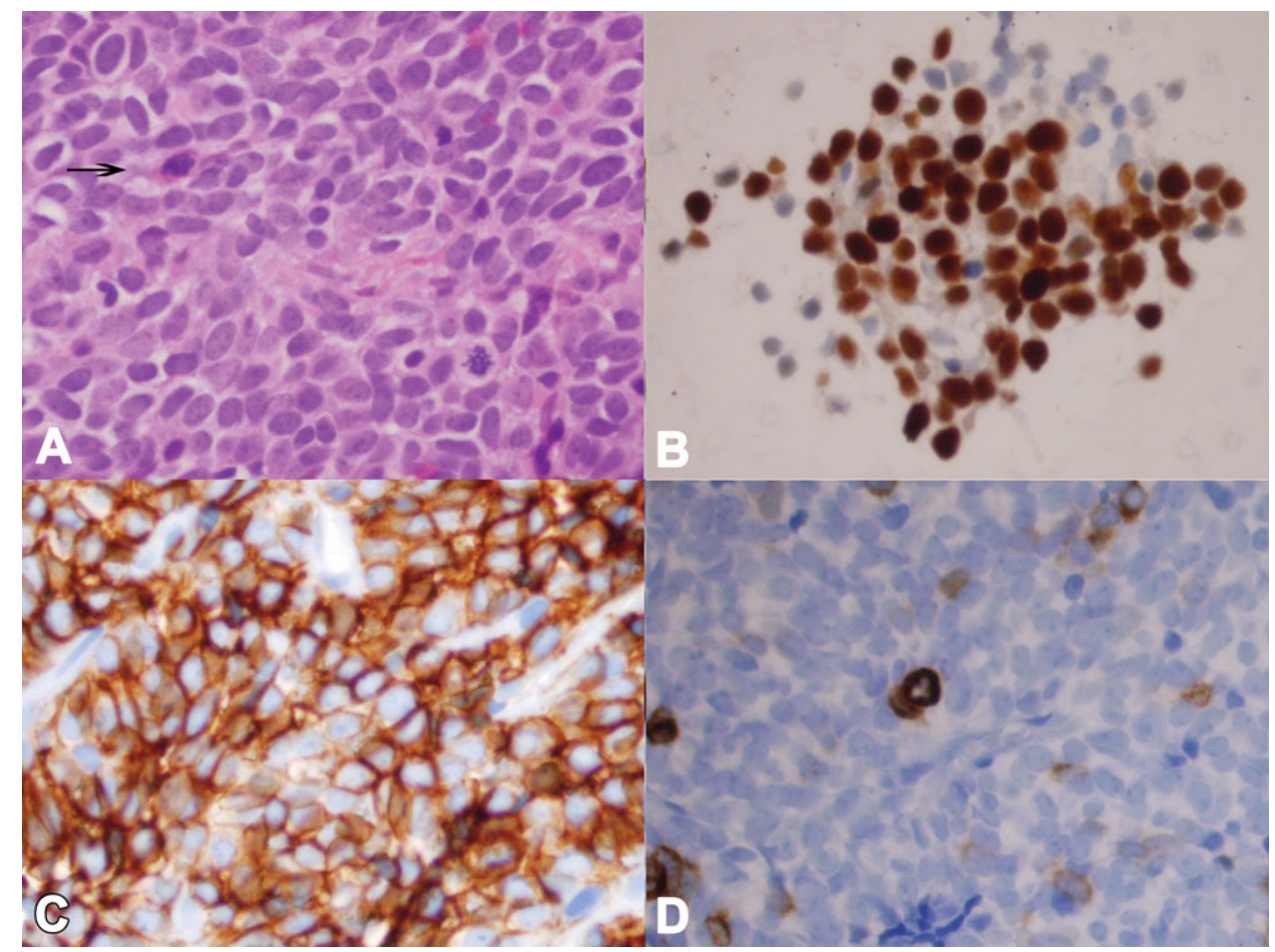

Fig. 4. H\&E stain of cell block section shows frequent mitotic figures and rare mast cells (arrow) (A). The malignant cells demonstrate diffusely strong nuclear staining for TLE-1 (B), membranous staining for CD56 (C) and focal positivity for pan-cytokeratin stain (D). 600×. [Color figure can be viewed in the online issue, which is available at wileyonlinelibrary.com.] 
FINE NEEDLE ASPIRATION OF PRIMARY MEDIASTINAL SS
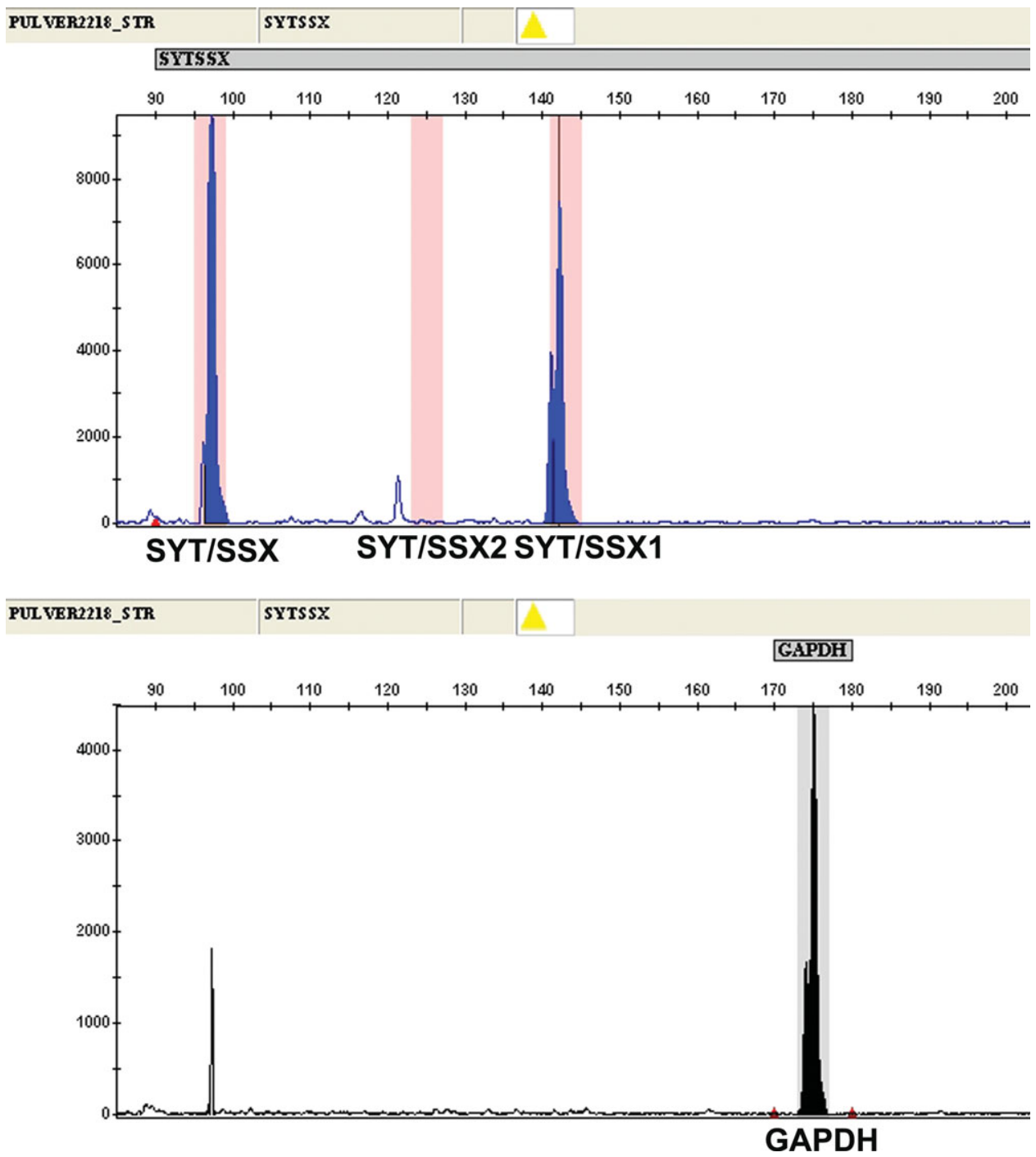

Fig. 5. Electropherogram demonstrates the presence of the SYT/SSX1 fusion gene transcript in this case. In the top panel, the peaks represent the SYT/SSX common bin and the SYT/SSX1 bin. The transcript of SYT/SSX2 is not amplified. The GAPDH peak in the bottom panel demonstrates that the nucleic acid extracted from the patient's sample was amplifiable. [Color figure can be viewed in the online issue, which is available at wileyonlinelibrary.com.]

reported in a large series. ${ }^{8}$ In a study of 60 cases of primary SS of the thorax (pulmonary, pleural and mediastinal), only five cases $(9 \%)$ were of mediastinal origin. ${ }^{9}$ Clinically, primary mediastinal SS has a male predilection, with age ranging from 3 to 83 years (median 35 years). Seventy percent of patients present at $20-50$ years of age. ${ }^{8}$ Chest pain and shortness of breath are the most common presenting symptoms. Other common symptoms include cough, pleural effusion, back pain, fever, anemia, and weight loss. ${ }^{8,9}$ The mass is usually large, ranging from 5 to $20 \mathrm{~cm}$. Primary mediastinal and pulmonary SS are aggressive tumors sharing common histologic features with soft tissue SS. ${ }^{8,9,14-16}$ The clinical features in our case are consistent with reports in the literature.

FNA cytomorphology of soft tissue SS has been well described. ${ }^{17}$ Cytologic diagnosis of soft tissue synovial sarcoma is usually straightforward by its characteristic cytomorphology and classic clinical presentation. FNAs of SS are typically hypercellular consisting of cells with round to oval nuclei and very high N/C ratios. The nuclei are usually uniform, bland appearing with finely granular chromatin and a small nucleolus. The cells are arranged in cohesive clusters and individually scattered. Other features described in the literature include the presence of nucleoli, vascular stalks, mast cells, lack of myxoid stroma, and occasional formation of tubules that can be mistaken for rosettes. Nuclear pleomorphism and abundant mitotic figures can be seen in poorly differentiated SS. ${ }^{11}$

In our patient, given the significant smoking history and location in the thoracic region coupled with the cyto- 
logic findings, our primary differential diagnoses included pulmonary neuroendocrine neoplasms such atypical carcinoid and small cell carcinoma, as well as basaloid squamous cell carcinoma which can all present with a centrally located mass in a smoker.

Pulmonary neuroendocrine tumors represent $\sim 25 \%$ of primary malignancies, and often arise from the lung adjacent to mediastinum. It can present as a mediastinal mass by direct tumor extension from the lung or by lymph node metastasis. Pulmonary neuroendocrine tumors include typical carcinoid, atypical carcinoid, small cell carcinoma, and large cell carcinoma. Morphologically, atypical carcinoid and small cell carcinoma are the main entities to be considered in this case. Smears of atypical carcinoids consist of single cells admixed with loosely cohesive cell clusters, rosette-like structures, and branching capillaries. ${ }^{18}$ The atypical carcinoid cells are round, plasmacytoid, or elongated, small to medium in size, with scant to moderate cytoplasm. Spindle cells can be seen. Mild to moderate nuclear atypia with coarsely granular chromatin and often prominent nucleoli are characteristic features. Nuclear molding and mitoses are mild to moderate. ${ }^{19,20}$ Presence of individual tumor cell necrosis is frequent. FNA smears of small cell carcinoma usually consist of predominantly streaming single cells with loose clusters demonstrating prominent nuclear molding, abundant mitoses, marked necrosis, apoptotic bodies, and nuclear crush artifact. Nucleoli are small and inconspicuous. Some of the cytologic features seen in atypical carcinoid and small cell lung carcinoma were present in our case, including occasional trabecular and pseudoacinar cell arrangement, spindle cells, scant cytoplasm with high N/C ratio, inconspicuous nucleoli, and readily identifiable mitotic figures. However, in our case, there was not a prominence of nuclear molding and the nuclear chromatin was finely granular rather than coarsely granular. In addition, the background was clean without significant necrosis, apoptotic bodies, and nuclear crush artifact typically seen in small cell lung carcinoma. IHC studies may be helpful as pulmonary neuroendocrine neoplasms are typically positive for cytokeratin, TTF-1, CD56, chromogranin, synaptophysin, and Ki-67 is near 100\% in small cell carcinomas.

Basaloid squamous cell carcinoma is an aggressive variant of squamous cell carcinoma that has a predilection for the upper aerodigestive tract, but can occur primarily in the lung in smokers. It usually begins centrally in the larger proximal bronchi. FNA smears consist of uniform cells with scant cytoplasm. The nuclei are hyperchromatic and the nucleoli are typically small and inconspicuous. There is a predominance of tightly cohesive tumor cell clusters with peripheral palisading and occasional dispersed cells. ${ }^{21}$ Nuclear molding and necrosis can be seen as well as abundant mitoses. Basaloid squamous cell carcinomas are typically positive for p63 and cytokeratin 5/6 immunostains while SS are negative.
Other neoplasms to consider in the mediastinum, which are not associated with smoking, include primary nonHodgkin's lymphoma, spindle thymoma, and Ewing sarcoma/primitive neuroectodermal tumors (PNET). Primary mediastinal large B-cell lymphoma and lymphoblastic lymphoma are two primary mediastinal lymphomas that often present as a mediastinal mass with compression effects. Primary mediastinal large B-cell lymphoma is a diffuse large B-cell lymphoma arising from thymic Bcells in the mediastinum. It accounts for $2-4 \%$ of the lymphomas, and is primarily seen in young adult females. Smears consist of predominantly large atypical cells with moderate amounts of cytoplasm, vesicular nuclei, and prominent nucleoli. Other cell types such as centroblasts, immunoblasts, or anaplastic cells are often present. Lymphoblastic lymphoma most commonly affects adolescents and young adults, but can be seen in any age group. Smears consist of a monotonous population of cells, which are medium in size with scant cytoplasm, round or convoluted nuclei, finely granular chromatin, inconspicuous nucleoli, and variable mitotic figures. ${ }^{22,23}$ In contrast to SS, smears of lymphoma usually consist of predominantly dispersed single cells. Cohesive clusters and nuclear molding are not predominant features. A background of lymphoglandular bodies, best seen on DiffQuik stained smears, is a clue for lymphoid origin of the tumor. Further classification of these lymphomas relies on IHC, flow cytometry and cytogenetic/molecular studies.

Thymomas are the most common primary epithelial neoplasm to arise in the anteriosuperior mediastinum. Although most patients are asymptomatic with the mass detected incidentally on chest imaging, others present with symptoms attributable to mass effects and paraneoplastic syndromes such as myasthenia gravis. Thymomas can occur in any age group, but particularly in the fifth and sixth decades with slight female predominance. The FNA smear is usually cellular with a mixture of bland epithelial cells and polymorphic lymphocytes in varying proportions giving rise to the classification into four major categories: (1) epithelial predominant; (2) lymphocyte predominant; (3) mixed lymphoepithelial; and (4) spindle cell types. In the spindled cell type, the tumor cells are elongated and can be found in interlacing fascicles with arborizing capillaries mimicking a mesenchymal neoplasm. ${ }^{24}$ The nuclei have finely dispersed and pale-stained chromatin with small and inconspicuous nucleoli. These features are similar to those in SS. A second population of polymorphic lymphocytes on the smear suggests thymoma. However, it can be diagnostically challenging if the smear consists of predominantly epithelial or spindle cells. IHC studies may be helpful as the epithelial component is diffusely positive for cytokeratin and is usually positive for CD20 and CD57, while the background lymphocytes are positive for TdT. 
Primary mediastinal Ewing Sarcoma/PNET is extremely rare and occurs predominantly in the pediatric population with a predilection for the posterior mediastinum. ${ }^{25}$ Smears of Ewing Sarcoma/PNET consist of a monotonous population of small round blue cells with scant cytoplasm. Nuclear molding is a constant and dominant feature. Fine wispy unipolar cytoplasmic tails can occasionally be seen. Spindle cells and rosette formation is not uncommon. ${ }^{26}$ IHC studies are not helpful in differentiating Ewing Sarcoma/PNET from SS as there is significant overlap. Molecular studies to identify the classic translocations are required for definitive diagnosis.

IHC studies are often used in the diagnosis of small round blue cell tumors in the mediastinum. SS is positive for cytokeratin, EMA, CD99, Bcl-2, and CD56. Bcl-2 and CD56 are very sensitive markers for SS, both of which are positive in up to $100 \%$ of cases of primary pulmonary and mediastinal SS. ${ }^{9}$ TLE-1, which shows positive nuclear staining in $96-97 \%$ of SS, has a strong correlation with the $\mathrm{t}(\mathrm{X} ; 18)$ translocation and is a more specific marker for $\mathrm{SS}^{27,28}$ Neuroendocrine tumor markers such as CD56, chromogranin, and synaptophysin are useful in identifying atypical carcinoids and small cell carcinomas. However, SS are also positive for CD56 in almost $100 \%$ of cases. ${ }^{9}$ Basaloid squamous cell carcinomas are positive for p63 and CK $5 / 6$ while SS are negative for both. Lymphomas are positive for CD45 while SS are negative. For the classification of lymphomas, the aspirated material should be triaged for flow cytometry during on-site evaluation if lymphoma is suspected. In thymomas, the epithelial component is diffusely positive for cytokeratin and usually expresses CD20 and CD57, while the lymphocytes are positive for TdT. Membranous staining of CD99 is useful in the diagnosis of Ewing Sarcoma/PNET; however, this can also be seen in SS. Therefore, molecular or cytogenetic studies are essential for definitive diagnosis.

In conclusion, we report an example of a primary mediastinal SS in the clinical setting of a long term smoker where carcinomas such as small cell carcinoma or basaloid squamous cell carcinoma would be more common. Including SS in the differential diagnosis of mediastinal masses along with familiarity with the cytologic features as well as understanding the value and limitations of ancillary tests are critical in ensuring an accurate diagnosis.

\section{References}

1. Wright PH, Sim FH, Soule EH, Taylor WF. Synovial sarcoma. J Bone Joint Surg Am 1982;64:112-22.

2. Schmidt D, Thum P, Harms D, Treuner J. Synovial sarcoma in children and adolescents. A report from the Kiel Pediatric Tumor Registry. Cancer 1991;67:1667-72.

3. Machen SK, Easley KA, Goldblum JR. Synovial sarcoma of the extremities: A clinicopathologic study of 34 cases, including semiquantitative analysis of spindled, epithelial, and poorly differentiated areas. Am J Surg Pathol 1999;23:268-75.
4. Fetsch JF, Meis JM. Synovial sarcoma of the abdominal wall. Cancer 1993;72:469-77.

5. Roth JA, Enzinger FM, Tannenbaum M. Synovial sarcoma of the neck: A followup study of 24 cases. Cancer 1975;35:124353.

6. Shmookler BM. Retroperitoneal synovial sarcoma. A report of four cases. Am J Clin Pathol 1982;77:686-91.

7. Fatimi SH, Anees A, Hanif HM, Muzaffar M. Synovial sarcoma of the lung presenting late with compression of mediastinal structures and its successful resection: A case report. Heart Lung Circ 2011;20:136-8

8. Suster S, Moran CA. Primary synovial sarcomas of the mediastinum: A clinicopathologic, immunohistochemical, and ultrastructural study of 15 cases. Am J Surg Pathol 2005;29:56978.

9. Hartel PH, Fanburg-Smith JC, Frazier AA, et al. Primary pulmonary and mediastinal synovial sarcoma: A clinicopathologic study of 60 cases and comparison with five prior series. Mod Pathol 2007; 20:760-9.

10. Jeganathan R, Davis R, Wilson L, McGuigan J, Sidhu P. Primary mediastinal synovial sarcoma. Ulster Med J 2007;76:10911.

11. Akerman M, Ryd W, Skytting B. Fine-needle aspiration of synovial sarcoma: Criteria for diagnosis: Retrospective reexamination of 37 cases, including ancillary diagnostics. A Scandinavian Sarcoma Group study. Diagn Cytopathol 2003;28:232-8.

12. Akerman M, Willen H, Carlen B, Mandahl N, Mertens F. Fine needle aspiration (FNA) of synovial sarcoma-a comparative histological-cytological study of 15 cases, including immunohistochemical, electron microscopic and cytogenetic examination and DNA-ploidy analysis. Cytopathology 1996;7:187-200.

13. Guillou L, Coindre J, Gallagher G, et al. Detection of the synovial sarcoma translocation $\mathrm{t}(\mathrm{X} ; 18)$ (SYT;SSX) in paraffin-embedded tissues using reverse transcriptase-polymerase chain reaction: A reliable and powerful diagnostic tool for pathologists. A molecular analysis of 221 mesenchymal tumors fixed in different fixatives. Hum Pathol 2001;32:105-12.

14. Begueret H, Galateau-Salle F, Guillou L, et al. Primary intrathoracic synovial sarcoma: A clinicopathologic study of $40 \mathrm{t}(\mathrm{X} ; 18)$-positive cases from the French Sarcoma Group and the Mesopath Group. Am J Surg Pathol 2005;29:339-46.

15. Essary LR, Vargas SO, Fletcher CD. Primary pleuropulmonary synovial sarcoma: Reappraisal of a recently described anatomic subset. Cancer 2002;94:459-69.

16. Okamoto S, Hisaoka M, Daa T, Hatakeyama K, Iwamasa T, Hashimoto H. Primary pulmonary synovial sarcoma: A clinicopathologic, immunohistochemical, and molecular study of 11 cases. Hum Pathol 2004;35:850-6.

17. Kilpatrick SE, Teot LA, Stanley MW, Ward WG, Savage PD, Geisinger KR. Fine-needle aspiration biopsy of synovial sarcoma. A cytomorphologic analysis of primary, recurrent, and metastatic tumors. Am J Clin Pathol 1996;106:769-75.

18. Anderson C, Ludwig ME, O'Donnell M, Garcia N. Fine needle aspiration cytology of pulmonary carcinoid tumors. Acta Cytol 1990;34:505-10

19. Mitchell ML, Parker FP. Capillaries. A cytologic feature of pulmonary carcinoid tumors. Acta Cytol 1991;35:183-5.

20. Szyfelbein WM, Ross JS. Carcinoids, atypical carcinoids, and small-cell carcinomas of the lung: Differential diagnosis of fine-needle aspiration biopsy specimens. Diagn Cytopathol 1988:4:1-8.

21. Marks RA, Cramer HM, Wu HH. Fine-needle aspiration cytology of basaloid squamous cell carcinoma and small cell carcinoma-A comparison study. Diagn Cytopathol 2011. doi: 10.1002/ dc. 21853 .

22. Jacobs JC, Katz RL, Shabb N, el-Naggar A, Ordonez NG, Pugh W. Fine needle aspiration of lymphoblastic lymphoma. A multiparameter diagnostic approach. Acta Cytol 1992;36:887-94. 


\section{HUANG ET AL.}

23. Wakely PE, Jr., Kornstein MJ. Aspiration cytopathology of lymphoblastic lymphoma and leukemia: The MCV experience. Pediatr Pathol Lab Med 1996;16:243-52.

24. Ali SZ, Erozan YS. Thymoma. Cytopathologic features and differential diagnosis on fine needle aspiration. Acta Cytol 1998;42:845-54.

25. Manduch M, Dexter DF, Ellis PM, Reid K, Isotalo PA. Extraskeletal Ewing's sarcoma/primitive neuroectodermal tumor of the posterior mediastinum with $\mathrm{t}(11 ; 22)(\mathrm{q} 24 ; \mathrm{q} 12)$. Tumori 2008;94:888-91.

26. Klijanienko J, Couturier J, Bourdeaut F, et al. Fine-needle aspiration as a diagnostic technique in 50 cases of primary Ewing sarcoma/pe- ripheral neuroectodermal tumor. Institut Curie's experience. Diagn Cytopathol 2012;40:19-25.

27. Terry J, Saito T, Subramanian S, et al. TLE1 as a diagnostic immunohistochemical marker for synovial sarcoma emerging from gene expression profiling studies. Am J Surg Pathol 2007;31: 240-6.

28. Knosel T, Heretsch S, Altendorf-Hofmann A, et al. TLE1 is a robust diagnostic biomarker for synovial sarcomas and correlates with $\mathrm{t}(\mathrm{X} ; 18)$ : Analysis of 319 cases. Eur J Cancer 2010; 46:1170-6. 